\title{
Are energetic electrons in the solar wind the source of the outer radiation belt?
}

\author{
Xinlin Li ${ }^{1}$ D. N. Baker, ${ }^{1}$ M. Temerin, ${ }^{2}$ D. Larson, ${ }^{2}$ R. P. Lin, ${ }^{2}$ \\ G. D. Reeves ${ }^{3}$ M. Looper, ${ }^{4}$ S. G. Kanekal,${ }^{5}$ and R. A. Mewaldt ${ }^{6}$
}

\begin{abstract}
Using data from WIND, SAMPEX (Solar, Anomalous, and Magnetospheric Particle Explorer), and the Los Alamos National Laboratory (LANL) sensors onboard geostationary satellites, we investigate the correlation of energetic electrons in the $20-200 \mathrm{keV}$ range in the solar wind and of high speed solar wind streams with relativistic electrons in the magnetosphere to determine whether energetic electrons in the solar wind are the source of the outer relativistic electron radiation belt. Though there is some correlation between energetic electron enhancements in the solar wind and enhancements in the outer radiation belt, the phase space density of $20-200 \mathrm{keV}$ electrons in the solar wind is not adequate to supply the outer radiation belt electrons. Although lower energy electrons in the solar wind could be a seed population of the outer radiation belt, such lower energy electrons cannot achieve relativistic energies through the normal process of radial transport which conserves the first adiabatic invariant. Thus additional internal acceleration processes are required within the magnetosphere to produce the outer radiation belt. High speed solar wind streams are well correlated with increased magnetic activity and with increased fluxes in the outer radiation belt. The maximum correlation between the high speed streams and the radiation belt flux occurs with an increasing time delay for higher energies and and lower $L$ values. We conclude that acceleration processes within the magnetosphere which are well correlated with high speed solar wind streams are responsible for the outer radiation belt electrons.
\end{abstract}

\section{Introduction}

The outer radiation belt consists of electrons with hundreds of $\mathrm{keV}$ to $\mathrm{MeV}$ energies. While there is no clear boundary between radiation belt electrons and lower energy plasmasheet and ring current electrons, above $0.4 \mathrm{MeV}$ electrons usually do not show rapid variability on the time scale of substorm injections [e.g., Baker et al. 1986]. Nevertheless, compared to the inner radiation belt, the outer zone shows a great deal of variability on a time scale correlated with magnetospheric storms and high speed solar wind streams [Paulikas and Blake, 1979]. Figure 1 is an example of radiation belt electrons measured by SAMPEX. The data, sorted according to L-value (in bins of $0.1 \mathrm{~L}$ ), are plotted ver-

${ }^{1}$ Lab. for Atmospheric and Space Physics, University of Colorado, Boulder

${ }^{2}$ Space Sciences Lab., University of California, Berkeley

${ }^{3}$ Los Alamos National Lab., Los Alamos, New Mexico

${ }^{4}$ Dept. of Space Sciences, Aerospace Corp., Los Angeles

${ }^{5}$ NASA GSFC and Hughes STX, Greenbelt, Maryland

${ }^{6}$ Calif. Inst. of Technology, Pasadena, California

Copyright 1997 by the American Geophysical Union.

Paper number 97GL00543.

0094-8534/97/97GL-00543\$05.00 sus day of year. Numerous examples of abrupt electron enhancements extend across all $\mathrm{L}$-values from $\mathrm{L} \sim 2$ to $\sim 7$.

The origin of the outer radiation belt electrons remains an unsolved problem. To understand the nature of the problem it is necessary to understand some basic properties of the outer radiation belt and the basic processes that are thought to be important in its dynamics. Three gradients typically characterize the outer radiation belt electrons. (1) At any given energy the phase space density, $f_{p}$, of electrons increases with decreasing $\mathrm{L}$ down to about $\mathrm{L}=4$ close to the so-called slot region ( $\mathrm{L}=2.5-3.5)$. (2) At any given $\mu$ (the first adiabatic invariant $\left.\mu=p_{\perp}^{2} / 2 m_{0} B\right)$, however, $f_{p}$ decreases with decreasing L. (3) At any given $\mathrm{L} f_{p}$ decreases with energy. None of these properties are absolutely true at all times but they are characteristic features le.g., Roederer, 1970].

Two processes that are important in the dynamics of the radiation belt are radial transport in the form of radial diffusion or impulsive injections and pitch angle scattering. Radial diffusion or impulsive injection conserves $\mu$ and thus inward transport increases the energy of the transported electrons. Pitch angle scattering approximately conserves the electron's energy while leading to electron loss by scattering into the loss cone and consequent precipitation into the atmosphere.

While radial transport can usually explain the increase in the electron flux at almost any $L$ and energy, it can not explain the origin of the outer radiation belt as a whole. Radial transport requires an outer boundary that is the ultimate source of the electrons but which is itself not explained by radial transport. Two obvious sources for the electron flux at such an outer boundary are electrons in the solar wind or electrons accelerated in the outer part of the magnetosphere. The purpose of this letter is to determine if energetic electrons in the solar wind are an adequate source of such electrons through the normal and understood process of radial transport, a process which conserves at least the first adiabatic invariant, or whether some other acceleration process, such as wave-particle interactions resulting in the heating of part of the electron distribution, is required within or at the boundaries of the magnetosphere. Since radial diffusion proceeds by moving electrons from regions of higher phase space density to lower phase space density and since losses occur during the process of radial diffusion due, for instance, to pitch angle scattering, for electrons in the solar wind to be the source of the outer radiation belt their phase space density should be substantially higher than that of the corresponding electrons in the radiation belt. In addition, we would then expect that there should be a correlation between changes in the solar wind flux and in the radiation belt.

It has been suggested that relativistic electrons in the solar wind, either from the Sun or from Jupiter, may be a source of the relativistic electrons in the magnetosphere [e.g., Baker et al., 1979; Baker et al., 


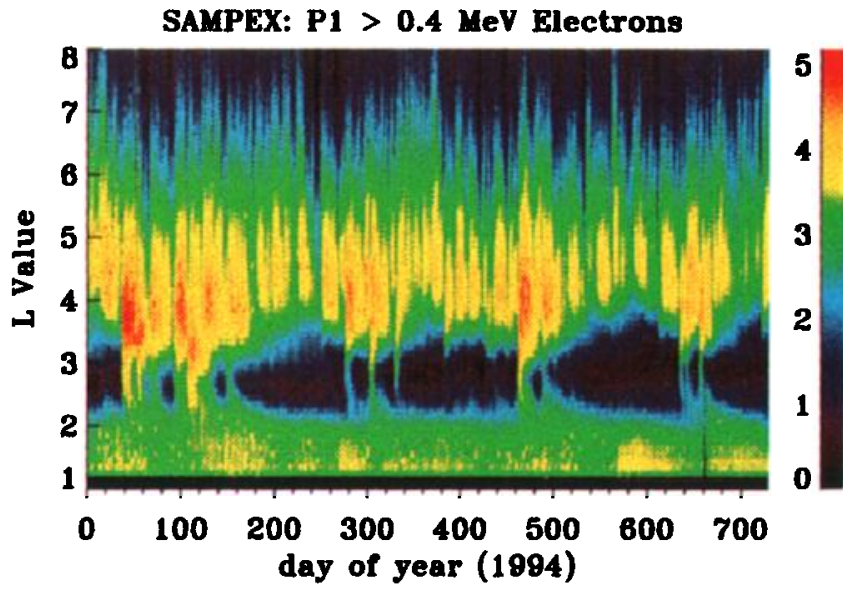

Figure 1. A color-coded representation of electron fluxes measured by the SAMPEX P1 channel $(>0.4$ $\mathrm{MeV}$ electrons and $>4 \mathrm{MeV}$ protons) from 01/01/1994 to $12 / 31 / 1995$. The data are binned in $0.1 \mathrm{~L}$-values and the range $1 \leq \mathrm{L} \leq 8$ is shown. Electrons dominate the count rate for $2 \leq L \leq 8$.

1986; Bezrodnykh et al., 1986]. However, Christon et al. [1989] showed that the outer radiation belt does not have the 13 month periodicity of the MeV Jovian electrons in the solar wind. It is more appropriate to compare electrons with tens to hundreds of $\mathrm{keV}$ in the solar wind with radiation belt electrons since such electrons would have about $\mathrm{MeV}$ energies after radially moving inward while conserving $\mu$ and their phase space density is larger. It has also been suggested that internal magnetospheric processes, which correlate with high speed solar wind streams, may be responsible for enhancements in the relativistic electrons [Paulikas and Blake, 1979; Baker et al., 1986; Chenette et al., 1988; Baker et al., 1994; Baker et al., 1996].

\section{Data}

We use data from WIND, SAMPEX, and the LANL sensors on geosynchronous satellites during the first half of 1995. The WIND electron fluxes in the energy range of $27-182 \mathrm{keV}$, the solar wind velocity, and solar wind density are provided by the 3D plasma and energetic particles experiment [Lin et al., 1995]; the magnetic field is from the magnetometer [Lepping et al., 1995]. SAMPEX was launched on 3 July 1992 into a nearly polar orbit with an altitude of about $600 \mathrm{~km}$, an inclination of $82^{\circ}$, and an orbital period of about 95 minutes [Baker et al., 1993]. The data here are daily averages binned at various invariant latitudes from the PET instrument [Cook et al., 1993], covering the energy range $>0.4 \mathrm{MeV}$ electrons, and 2-6 MeV electrons (ELO), and daily averaged electron data from the geosynchronous LANL sensors in the energy range of $0.7-1.8 \mathrm{MeV}$ and 1.8-3.5 MeV [Meier et al., 1996]. The first half of 1995 was in the declining phase of the solar cycle approaching sunspot minimum, a time when recurrent high speed solar wind streams emanating from persistent coronal holes were prominent. WIND and SAMPEX had continuous measurements of the solar wind and of relativistic electrons in the magnetosphere, respectively. The LANL data also had continuous coverage of relativistic electrons during this period.

\section{Observation and Discussion}

Figure 2 shows various solar wind and magnetospheric quantities for the first half of 1995. Panel (b) shows the recurrent high speed streams during the first half of 1995. These high speed streams are preceded by

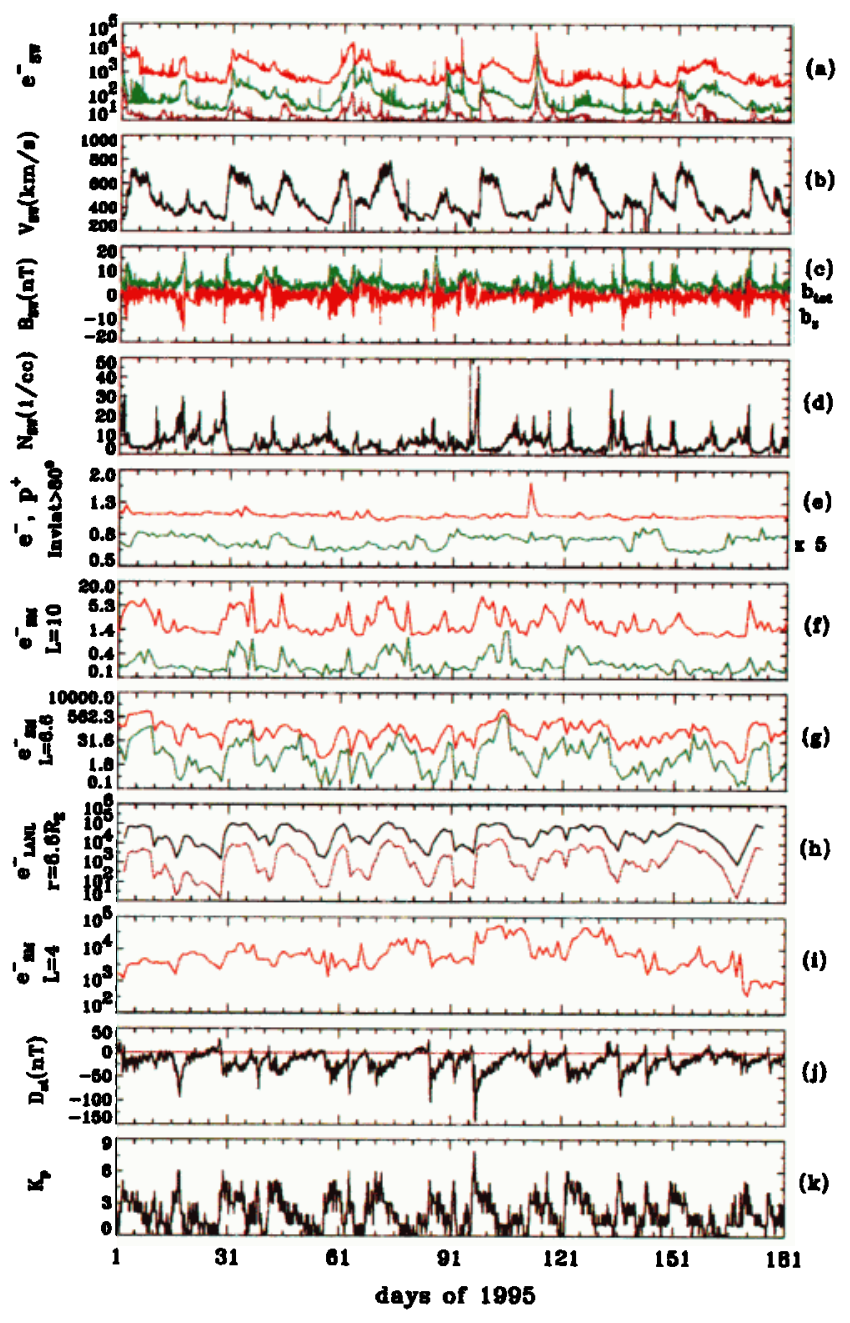

Figure 2. Various parameters plotted vs. time for the first half of 1995. The panels are: (a) differential flux of electrons (electron $/ \mathrm{cm}^{2}$-s-sr-MeV) in the solar wind (every 15 minutes) with the energies: $27.2 \mathrm{keV}$ (red), $66.3 \mathrm{keV}$ (green), and $182 \mathrm{keV}$ (purple); (b) solar wind velocity (every 14 minutes), $V_{s w}$; (c) the hourly averaged magnitude and $\mathrm{Bz}$ component of the interplanetary magnetic field; (d) solar wind plasma density (every 14 minutes), $N_{s w}$; (e), (f), (g), (i) daily averages of the integral flux of electrons (electron $/ \mathrm{cm}^{2}$-s-sr) measured by SAMPEX, red (green) line is for $>0.4 \mathrm{MeV}$ (2-6 MeV) electrons respectively, for invariant latitude greater than $80^{\circ}, \mathrm{L}=10, \mathrm{~L}=6.6, \mathrm{~L}=4$; The $>0.4 \mathrm{MeV}$ electron channel also responds to $>4 \mathrm{MeV}$ protons; for invariant latitude $\geq 80^{\circ}$ it is dominated by cosmic ray protons, while for $\overline{\mathrm{L}}=4,6.6$, and 10 it is dominated by trapped electrons. (h) daily averages of the differential flux (electron $/ \mathrm{cm}^{2}$-s-sr-MeV) of electrons measured by the LANL sensor onboard geosynchronous satellite (1989-046) with the energies: 0.7-1.8 MeV (black) and 1.8-3.5 MeV (purple). The last two panels $((\mathrm{j})$ and $(\mathrm{k}))$ display $D_{s t}$ and the $K_{P}$ indices. 
magnetic field enhancements and solar wind density (panels (c) and (d)). The high speed streams together with their accompanying density and magnetic field enhancements increase magnetospheric activity as indicated by decreases in Dst $(\operatorname{panel}(j))$ and increases in $\mathrm{Kp}$ (panel(k)). Some of the energetic solar wind electron fluctuations (panel (a)) are correlated with the solar wind speed such as on days $28,55,97$, and 148 (the numerous short time-scale spikes are believed to be electrons accelerated by the Earth's bow shock [Lin et al., 1996]). However, at other times there are no or only weakly correlated enhancements such as on days 68 and 112 .

Energetic interplanetary electrons $(>0.4 \mathrm{MeV})$ should appear directly in the polar cap and be seen by SAMPEX (panel (e)). Because of the relatively low level of energetic electron flux and high background due to cosmic ray protons which dominate in the high latitude region, only the day 112 solar particle event is clearly seen by SAMPEX in the $>0.4 \mathrm{MeV}$ electron channel in the polar cap. More energetic electrons, including Jovian electrons, are seen in the $2-6 \mathrm{MeV}$ channel. The relatively low level of such electrons in the solar wind as reflected in the polar cap fluxes can be compared directly with the much higher radiation belt levels (panels (f) through (i)). Note that the $2-6 \mathrm{MeV}$ flux is multiplied by 5 in panel (e). Panel (f) shows the flux at the foot of field line at $\mathrm{L}=10$, which is near the limit of stably trapped electrons. Panels $(\mathrm{g})$ and $(\mathrm{h})$ show electrons near the geosynchronous magnetic field line at $\mathrm{L}=6.6$, (g) from SAMPEX and (h) from LANL detectors at geosynchronous orbit. The $L$ values at SAMPEX are determined by mapping the field line using the International Geomagnetic Reference Field (IGRF) model 1990 extrapolated to the time of observation. The flux at SAMPEX is about 2-3 orders of magnitude lower than the corresponding LANL fluxes because SAMPEX measures only electrons near the loss cone. However, the temporal variations at SAMPEX and geosynchronous are well correlated despite this and despite the differences in the energy ranges of the respective detectors. Panel (i) shows >0.4 MeV electron flux at the foot of the field line near the usual peak of the outer zone flux at $\mathrm{L}=4$. At fluxes $>10^{4} / \mathrm{cm}^{2}-\mathrm{s}-\mathrm{sr}-\mathrm{MeV}$, this channel is subject to saturation but the temporal structure of the fluxes can still be discerned.

We first compare the phase space density of electrons measured in the solar wind and in the magnetosphere. The known parameter for electrons is the differential flux. The phase space density, $f_{p}$, and differential flux, $j$, are related by

$$
f_{p}=j / p^{2}
$$

where $p\left(=m_{0} \gamma v\right)$ is the relativistic electron momentum. The average differential flux of $0.7-1.8 \mathrm{MeV}$ in panel (h) for this period is about 43000 electron $/ \mathrm{cm}^{2}$ -

Table 1. Comparison of $j_{s w}$ in solar wind at different energies with $j\left(=160385\right.$ electrons $/ \mathrm{cm}^{2}$-s$\mathrm{sr}-\mathrm{MeV}$ ) at $0.7 \mathrm{MeV}$ at geostationary orbit.

\begin{tabular}{lcc}
\hline$j=f_{p} * p^{2}$ & required $j_{s w}$ & measured $j_{s w}$ \\
\hline $27.2 \mathrm{keV}$ & & \\
$66.3 \mathrm{keV}$ & 3797 & 1470 \\
$182 \mathrm{keV}$ & 9604 & 142 \\
& 29161 & 21 \\
\hline
\end{tabular}
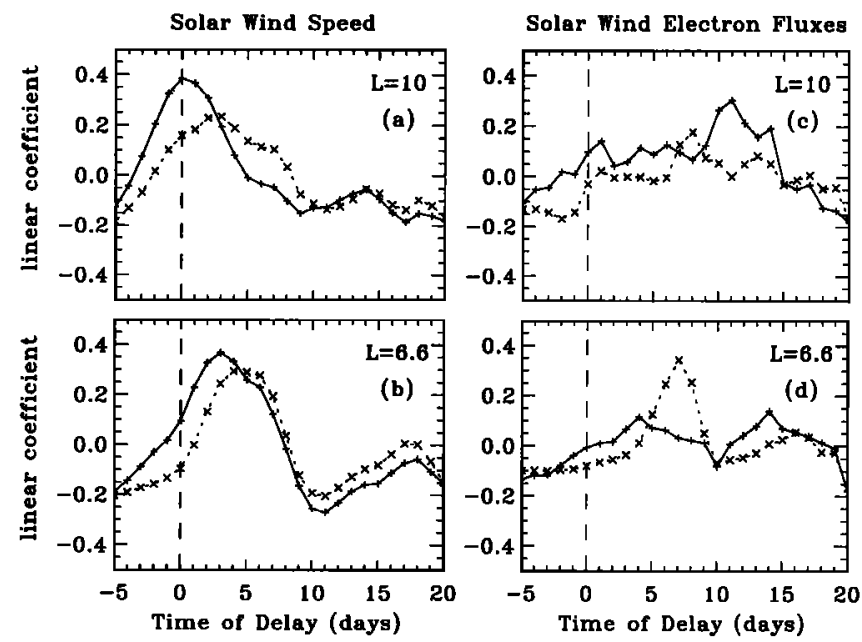

Figure 3. Daily averaged solar wind speed vs. SAMPEX electron fluxes (solid for $>0.4 \mathrm{MeV}$ and dotted for $2-6 \mathrm{MeV}$ ) at $\mathrm{L}=10$ (a) and $\mathrm{L}=6.6(\mathrm{~b})$. Daily averaged solar wind electron fluxes vs. SAMPEX electron fluxes at $\mathrm{L}=10$ (c) and $\mathrm{L}=6.6$ (d), solid for $27.2 \mathrm{keV}$ vs. $>0.4$ $\mathrm{MeV}$ and dotted for $182 \mathrm{keV}$ vs. 2-6 MeV.

s-sr-MeV. The differential flux at $0.7 \mathrm{MeV}$ is estimated to be $1.6 \times 10^{5}$ electron $/ \mathrm{cm}^{2}$-s-sr-MeV using a power law to fit the energy spectra $\left(j=A E^{-3}\right)$. If the solar wind electrons in panel (a) are the source of geosynchronous electrons in panel $(h)$, the differential fluxes in panel (a) should exceed a minimum level. The required and actually measured differential fluxes (electron $/ \mathrm{cm}^{2}$ $\mathrm{s}$-sr- $\mathrm{MeV}$ ) of $27.2 \mathrm{keV}, 66.3 \mathrm{keV}$, and $182 \mathrm{keV}$ during this period are listed in Table 1. An electron with a perpendicular energy of $0.7 \mathrm{MeV}$ at geosynchronous orbit in a magnetic field of $120 \mathrm{nT}$ corresponds to electrons with perpendicular energies covered by $27.2 \mathrm{keV}, 66.3 \mathrm{keV}$, and $182 \mathrm{keV}$ energy channels in magnetic fields of 3-20 $\mathrm{nT}$, which are in the range of the solar wind magnetic fields shown in panel (c) of Fig. 2. The average magnitude of the solar wind magnetic field for this period is $5.25 \mathrm{nT}$, so on average an electron with a perpendicular energy of $0.7 \mathrm{MeV}$ in a magnetic field of $120 \mathrm{nT}$ corresponds to a solar wind electron of $49.2 \mathrm{keV}$. Comparing the numbers, the electrons measured in the solar wind are not adequate to explain the electrons measured at geostationary orbit even without taking into account any losses that may occur during radial transport.

There are other significant correlations between quantities in the solar wind and electrons in the magnetosphere. Panel (f) and (g) of Fig. 2 show similar temporal variations in the electron flux. The electron flux enhancements are correlated with the the solar wind speed (panel (b)) with some delay. Electron enhancements appear to occur earlier at larger L-shells and lower energies following enhancements in the solar wind speed. To demonstrate these effects, the linear correlation coefficient for different time delays between the daily averaged solar wind speed and the SAMPEX electron flux at $\mathrm{L}=10$ and $\mathrm{L}=6.6$ is shown in panel (a) and (b) of Fig. 3. Panel (a) shows that the $>0.4 \mathrm{MeV}$ electrons at $\mathrm{L}=10$ correlate best with the solar wind speed on the same day while 2-6 MeV electrons correlate best with the solar wind speed after a two or three day delay. Panel (b) shows that the $>0.4 \mathrm{MeV}$ electrons at $\mathrm{L}=6.6$ correlate best with the solar wind speed after a three 
day delay while 2-6 MeV electrons correlate best with the solar wind speed after a four day delay.

The linear correlation coefficient for different time delays between the daily averaged solar electrons and electrons in the magnetosphere measured by SAMPEX at $\mathrm{L}=10$ and $\mathrm{L}=6.6$ are plotted in panel (c) and (d) of Fig. 3. In general the correlations between solar wind electrons and magnetospheric electrons are difficult to interpret and may be dominated by a few larger events. Further study is required to see if there is any correlation that is not explained by the correlation between the high speed streams and magnetospheric electrons and the correlation between the high speed streams and solar wind electrons. In any case, the phase space density of the $182 \mathrm{keV}$ electrons is too small to be a source of the 2-6 MeV electrons at $\mathrm{L}=6.6$. We obtain a differential flux 6043 (electron/ $\mathrm{cm}^{2}$-s-sr-MeV) at $2 \mathrm{MeV}$ using a power law with power index of three to fit the electrons in panel $(\mathrm{h})$ of Fig. 2, the required differential flux of $182 \mathrm{keV}$ electron according to equation (1) should be at least $1 / 28$ of 6043 or 216 (electron $/ \mathrm{cm}^{2}-\mathrm{s}-\mathrm{sr}-\mathrm{MeV}$ ). In fact, the average differential flux of $182 \mathrm{keV}$ is only 21 (electron $/ \mathrm{cm}^{2}$-s-sr-MeV), less than $10 \%$ of the required value.

\section{Conclusions}

Although the fluctuations in the $>20 \mathrm{keV}$ electrons in the solar wind at first suggested a possible direct link between these electrons and the outer radiation belt, the insufficient phase space density is an unambiguous argument, which leads us to conclude that $>20 \mathrm{keV}$ electrons in the solar wind are not an adequate source of the outer radiation belt through the normal and understood process of radial transport. The more numerous lower energy electrons in the solar wind require additional acceleration in order to be the source of the radiation belt. Thus we conclude that acceleration processes within the magnetosphere, which are well correlated, at least during the time period of our study, with high speed solar wind streams, are responsible for the outer radiation belt electrons. In addition we find that that the correlation of the outer radiation belt electrons with solar wind speed occurs with a delay that increases with the energy and smaller radial distance of the outer radiation belt electrons. It is important to further correlate energetic electron enhancements in the magnetosphere with various combination of solar wind parameters to determine the solar wind conditions most responsible for the enhancement of outer radiation belt electrons. A more quantitative study of such is being undertaken.

Acknowledgments. We thank R. Lepping for providing WIND magnetic field data. Programming effort by Steve Monk is acknowledged. The work at University of Colorado was supported by NASA grant NAG5-2681, NAGW-5152, and NSF grant ATM-9624390. The work at University of California at Berkeley was supported by NASA grant NAG52815, NAGW-1098, NAG5-3198, and NSF grant ATM-9224688. The work at LANL was supported by DoE of Basic Energy Science. The work at the Aerospace Corporation was supported by NASA under Cooperative Agreement 26979B.
The work at Calif. Inst. of Technology was supported by NASA grant NAS5-30704.

\section{References}

Baker, D. N., P. R. Higbie, R. D. Belian, and E. W. Hones, Do Jovian electrons influence the terrestrial outer radiation zone? J. Geophys. Res., 6, 531, 1979.

Baker, D. N., et al., Highly relativistic electrons in the earth's outer magnetosphere, 1. Lifetimes and temporal history 1979-1984, J. Geophys. Res., 91, 4265, 1986.

Baker, D. N., et al., An overview of the Solar Anomalous and Magnetospheric Particle Explorer (SAMPEX) mission, IEEE Trans. on Geosci. and Remote Sensing, 31, $531,1993$.

Baker, D. N., et al., Relativistic electron acceleration and decay time scales in the inner and outer radiation belts: SAMPEX, Geophys. Res. Lett., 21, 409, 1994.

Baker, D. N., X. Li, J. B. Blake, and S. Kanekal, Strong electron acceleration in the Earth's Magnetosphere, COSPAR, Birmingham, Britain, 1996.

Bezrodnykh, I. P., $\in t$ al., Variations in the intensity of the relativistic electron flux at the orbit of a geostationary satellite, Cosmic Res., 24, 762, 1986.

Chenette, D. L., et al., How are geosynchronous electrons related to the jovian component ?, (abstract), Conf. Proc. 27th of the Plenary Meetings of COSPAR, 114, 1988.

Christon, S., et al., Relativistic electrons at geosynchronous orbit, interplanetary electron flux, and the 13-month jovian synodic year, Geophys. Res. Lett.16, 1129, 1989.

Cook, W. R., et al., PET: A proton/electron telescope for studies of magnetospheric, solar, and galactic particles, IEEE Trans. on Geosci. and Remote Sensing, 31, 565, 1993.

Lepping, R. P., et al. The WIND magnetic field investigation, The Global Geospace Mission, Space Science Rev., 71,207, 1995.

Lin, R. P., et al., Observation of an impulsive solar electron event extending down to $\sim 0.5 \mathrm{keV}$ energy, Geophys. Res. Lett., 23, 1211, 1996.

Lin, R. P., et al., A three-dimensional plasma and energetic particle investigation for the WIND spacecraft, Space Science Rev., 71, 125, 1995.

Meier, M. M., et al., The energy spectrometer for particles (ESP): Instrument description and orbital performance, AIP Conf. Proc. 383 on Earth's Trapped Particles Environment, Ed. by G. D. Reeves, page 203, 1996.

Paulikas, G. A., and J B. Blake, Effects of the solar wind on magnetospheric dynamics: Energetic electrons at the synchronous orbit, Quantitative Modeling of Magnetospheric Processes, 21, Geophys. Monograph Series, 1979.

Roederer, J. G., Dynamics of Geomagnetically Trapped Radiation, 1970, Springer, Heidelberg.

D. N. Baker and X. Li, LASP/CU, 1234 Innovation Dr., Boulder, CO 80303-7814; e-mail: lix@kitron.colorado.edu

D. Larson, R. P. Lin, and M. Temerin, SSL, University of California, Berkeley, CA 94720

G. D. Reeves, Mail Stop D-436, Los Alamos National

Laboratory, Los Alamos, NM 87545

M. Looper, Department of Space Sciences, Aerospace Corporation, Los Angeles, CA 90009-2957

S G Kanekal, NASA GSFC Code 690 and Hughes STX, Greenbelt, MD 20771

R. A. Mewaldt, Space Radiation Lab., Calif. Inst. of Technology, Pasadena, CA 91125

(Received October 29, 1996; accepted February 13, 1997.) 